\title{
Neurologic Manifestations of SARS-CoV-2, the Virus that Causes COVID-19
}

\author{
Hassan Naji
}

\section{ABSTRACT}

Coronaviruses have been associated with many outbreaks in the past and the recent pandemic caused by SARS-CoV-2 has spread around the globe due to its high transmission rate. SARS-CoV-2 has reported to be associated with respiratory and cardiac complications, but recent case series and case reports of COVID-19 patients suggest that it is also associated with neurological manifestations. The most commonly observed neurological manifestation are headache, anomia, ageusia, dizziness, delirium and the complications include Guillain-Barre syndrome (GBS), acute encephalitis, acute myelitis, and cerebrovascular disorders. The pathways leading to neuronal damage are the retrograde neuronal and hematogenous pathway. This paper is aimed to explain the neurological manifestations associated with COVID-19 patients and the possible pathophysiological mechanisms of neuronal invasion.

Keywords: 2019-nCoV, SARS-CoV-2, Coronavirus, Novel coronavirus, COVID-19, MERS-CoV, SARS-CoV, Receptor-binding domain, ACE2 receptors, Central nervous system, Neurologic manifestation, Hypogeusia, Hyposmia, Neuralgia, Impaired consciousness, Acute cerebral diseases, Acute hemorrhagic necrotizing encephalopathy, Transverse myelitis and encephalitis, Epilepsy, Guillain-Barre syndrome, Ataxia, Skeletal muscle injury.
Published Online: November 13, 2020

ISSN: $2593-8339$

DOI: $10.24018 /$ ejmed.2020.2.6.533

\section{Hassan Naji *}

Purdue University Northwest, USA. (e-mail: hnaji@pnw.edu)

*Corresponding Author

\section{INTRODUCTION}

People around the globe are suffering from a pandemic caused by COVID-19. The early reports of viral infection started in December 2019 in Wuhan, China. On February 11, 2020, World Health Organization (WHO) officially named the disease as COVID-19 and the viral strain as SARS-CoV-2. The pandemic has caused more than 2.3 million laboratory confirmed cases of COVID-19 including 162,000 deaths worldwide [9]. There are many human CoVs (HCoVs) known to exist and caused respiratory and enteric diseases, including OC43, NL63, 229E, HKU1, Middle East Respiratory Syndrome CoV (MERS-CoV), and Severe Acute Respiratory Syndrome CoV (SARS-CoV). MERS$\mathrm{CoV}$ and SARS-CoV are known to be associated with outbreaks in the past.

Like other HCoVs, the SARS-CoV-2 also belongs to the beta-coronaviride family. SARS-CoV-2 is an enveloped virus with 29,903 bp single-stranded, positive sense RNA genome. Taxonomically, SARS-CoV-2 belongs to the clade of bat-derived SARS-CoV and use the same angiotensinconverting enzyme 2 (ACE2) receptors to gain entry into the host cells. Additionally, similarities are also observed between the genomic sequence of SARS-CoV-2 and MERSCoV. However, MERS-CoV uses dipeptidyl peptidase 4 receptor to infect the host cells [1]. The common presenting symptoms of SARS-CoV-2 are also similar to SARS-CoV and MERS-CoV, including fever, fatigue, cough, myalgias and diarrhea that progress to acute respiratory distress syndrome (ARDS), pneumonia and shock [2]. However, recent studies have shown that SARS-CoV-2 also causes cardiac and neurological manifestations including headache, anosmia, acute necrotizing hemorrhagic encephalopathy, neuropsychiatric manifestations, ageusia and stroke [3]. A study of 214 severe cases of COVID-19 in Wuhan reported some common neurological manifestations like altered consciousness and strokes in severe cases of COVID-19. They reported that almost $36 \%$ (78 patients) of severely ill patients of COVID-19 developed neurological issues [4].

\section{ACE2 RECEPTORS AND NEUROLOGICAL MANIFESTATIONS IN COVID-19}

Studies have shown that ACE2 is the target cellular receptor for SARS-CoV-2 spike (S) protein. The spike protein S1of SARS-CoV-2 attaches with ACE2 receptor of the host cell membrane. After being internalized, the viral RNA starts translation and replication. Progeny viruses are released and the infection spreads in the body via circulation. ACE2 receptors are widely expressed in the 
lungs, kidneys, heart, intestines, and testicles. These receptors are also present in glial cells in the central nervous system (CNS). Hence, SARS-CoV-2 has the ability to infect the neuronal tissue in brain and spinal cord, causing neurological issues. The primary target of SARS-CoV-2 is the respiratory epithelium, and it spreads to the neuronal tissue via bloodstream. ACE2 receptors are also abundantly expressed on tongue, allowing binding of SARS-CoV-2, resulting in the damage of taste receptors that lead to ageusia [5].

Both SARS-CoV and SARS-CoV-2 enter the cell by interacting with ACE2 receptors. The binding affinity of the spike protein ectodomain of SARS-CoV-2 is $10-20$ times greater than that of SARS-CoV. The difference in the binding affinities with ACE2 receptors can be explained by the pairwise sequence alignments of receptor binding domain (RBD) subdomain-1 of SARS-CoV-2, a spike glycoprotein of bat-CoV RaTG13 and S1 protein, partial of SARS-CoV GD322. The comparison showed that the three coronaviruses are highly similar but they are not identical (Fig. 1) [6].

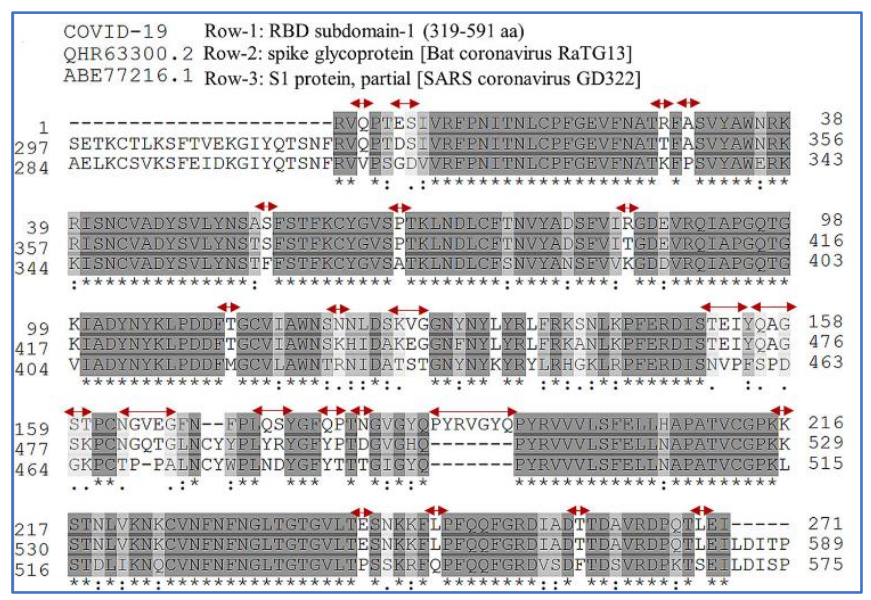

Fig. 1. Pairwise sequence alignment of receptor binding domain (RBD) subdomain-1 (319th-591st) amino acid) of SARS-CoV-2, a spike glycoprotein of bat-CoV RaTG13 and S1 protein, partial of SARS-CoV GD322 from BLASTp results. Horizontal arrows represent contrasting areas between the sequences. (Adopted from [6]).

\section{NEUROLOGICAL MANIFESTATIONS AND COMPLICATIONS OF COVID-19}

The typical symptoms of SARS-CoV-2 are fever, sore throat, cough, fatigue, diarrhea, and shortness of breath, with the disease progressing to respiratory and cardiac complications. However, it is being reported recently that SARS-CoV-2 causes neurological manifestations due to viral invasion in central nervous system (CNS). The neurological manifestations of the disease can be observed following recovery, or late in the disease, or even shortly after infection [7].

Past autopsies of SARS-CoV infected patients showed edema and neuronal degeneration of brain tissue. SARS$\mathrm{CoV}$ has also been isolated from brain tissue of the bodies which was further confirmed by in-situ hybridization, immunohistochemistry (IHC), and electron microscopy. As SARS-CoV and SARS-CoV-2 viruses share similar genomic sequence, it is likely that SARS-CoV-2 would follow similar pattern [8].
The neurological manifestations of SARS-CoV-2 include headache, dizziness, hypogeusia, hyposmia, seizures, neuralgia, impaired consciousness, acute cerebrovascular disease, acute hemorrhagic necrotizing encephalopathy, transverse myelitis and encephalitis and the complications involve epilepsy, Guillain-Barre syndrome, ataxia and skeletal muscle injury (Fig. 2). Headache and dizziness are the most frequently observed neurological manifestations. The earliest symptoms of neurological problems are loss of sense of taste and smell. The neurological manifestations and complications of SARS-CoV-2 are more common among severely-ill patients [9]. Another prospective study in France reported the most frequently observed neurological manifestations in COVID-19 patients at the time of discharge were agitation (69\%), confusion (65\%), corticospinal tract signs $(67 \%)$ and dysexecutive syndrome $(36 \%)$ [10]. It is believed that the cardio-respiratory diseases responsible for the death of SARS-CoV-2 infected patients may partially be attributed to brainstem dysfunction.

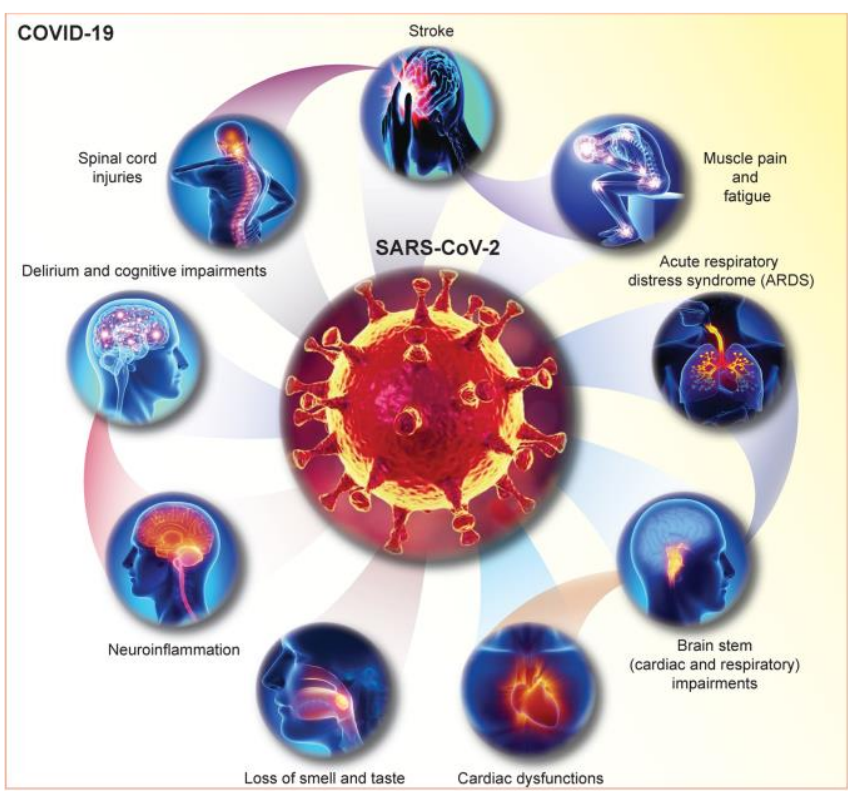

Fig. 2. Some neurological manifestations of COVID-19 [11].

Recent studies suggest that the acute respiratory distress syndrome (ARDS) observed in COVID-19 patients can also be related to viral damage to the neurons of brainstem. ARDS is the leading cause of mortality in COVID-19 patients suggesting that neuronal involvement in patients can also lead to respiratory complications [12]. Acute hemorrhagic necrotizing encephalopathy (ANE) is reported in a female patient in her late fifties. The magnetic resonance imaging (MRI) of her brain showed a rim of hemorrhagic enhancing lesions within the bilateral thalami, sub-insular regions and medial temporal lobes [13]. The acute myelitis observed in COVID-19 patients is attributed to the cytokine storm and hyperactive inflammatory response which is evident by increased levels of C-reactive protein, IL-6, serum ferritin and serum amyloid A. There are also reports, suggesting that COVID-19 patients experience convulsions in prevalent areas. The primary or secondary involvement of CNS in the patients infected with SARSCoV-2 can be determined by analysis of serum urea, electrolytes, creatinine, and concentration of blood gases 
(PO2 and PCO2). Recently, SARS-CoV-2 has been isolated from CSF of COVID-19 patients who develop neurological symptoms. In the previous outbreak of SARS-CoV, the virus was isolated from the CSF of infected patients. In order to save the patients of COVID-19 from developing further complications, it is necessary to establish an early differential diagnosis of neurological involvement [9].

\section{PATHOPHYSIOLOGY OF NEUROLOGICAL INVASION BY COVID-19}

Recent data suggests that SARS-CoV-2 is neurotropic as well as neurovirulent. Soon after infection, the virus invades central nervous system and enters into CSF, brain stem and cortex. The neuro-invasive ability of the virus is attributed to the expression of ACE2 receptors in neurons and endothelial cells. The spike protein of SARS-CoV-2 binds to ACE2 receptors and infects the cells [11]. It is suggested that SARS-CoV-2 can transfer to neuronal tissue via the hematogenous pathway or the retrograde axonal pathway across the cribriform plate or the olfactory epithelium (Fig. 3).

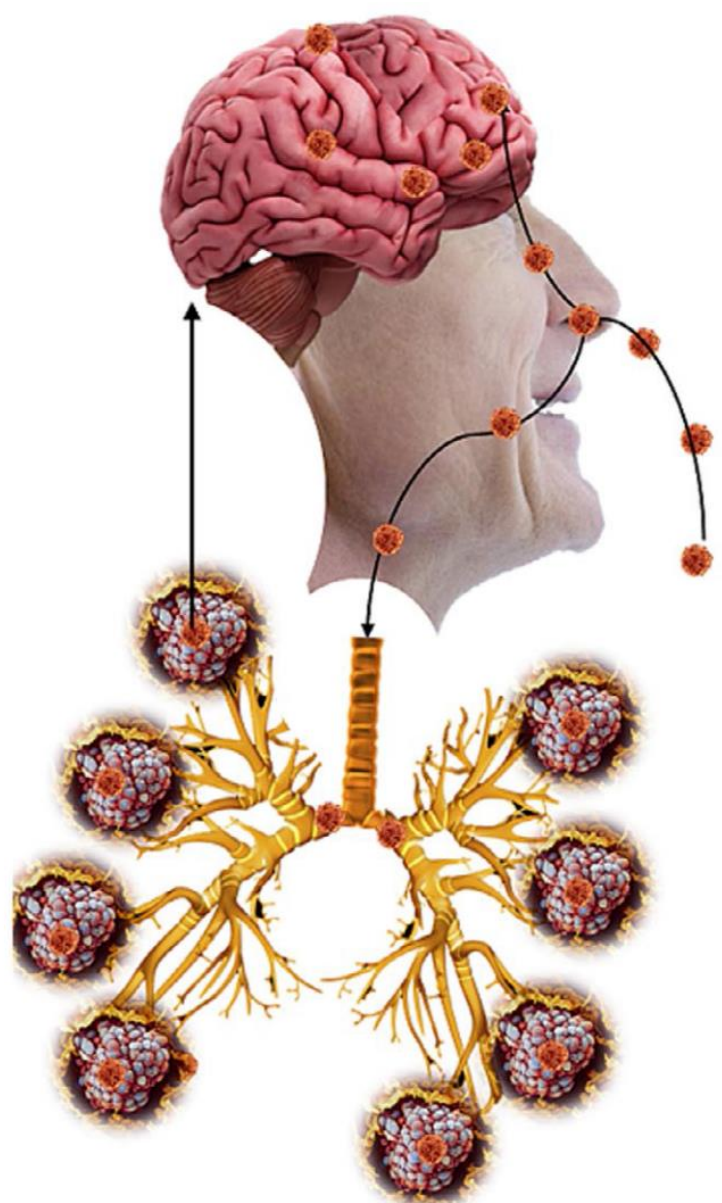

Fig. 3. Routes of entry of SARS-CoV-2 in the brain via hematogenous pathway or the cribriform plate (Adopted from [8]).

SARS-CoV-2 enters the cerebral circulation via the general circulation. The slow blood movement within the micro-circulation also enables the virus to interact with ACE2 receptors being expressed in the capillary endothelium. New viral particles bud from the endothelial cells and facilitate the entry of viral particles in the brain.
The virus crosses the blood-brain barrier and interacts with ACE2 receptors in the neurons and microglia. The replication of virus starts within the neuronal cells, damaging the blood-brain barrier. This initiates a cascade of inflammation within the brain with collateral damage, deliriums, secondary seizures, and stroke (Fig. 4). In addition to this damage, the rupture of capillary endothelium within the cerebral tissue is followed by bleeding, that also increases the mortality rate among COVID-19 patients [6].

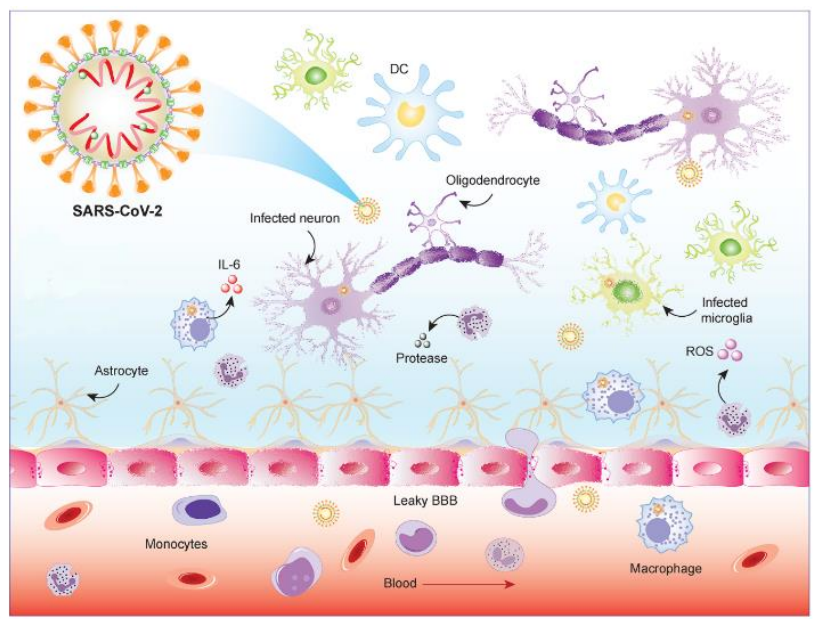

Fig. 4. Neurovirulence of SARS-CoV-2. BBB: blood-brain barrier, DC: dendritic cell, IL-6: Interleukin-6, ROS: reactive oxygen species. (Adopted from [11]).

Experiments on mice demonstrated that $\mathrm{CoV}$ enters the brain in seven days via retrograde pathway through cribriform bone of the ethmoid bone or the olfactory epithelium. This pathway of transfer can be considered in COVID-19 patients who exhibit anosmia and dysgeusia during the early phase of infection and later presented with neurological manifestations [8]. Previously, murine models showed that SARS-CoV and OC43-CoV enter the olfactory bulb via nasal cavity, followed by invasion of CNS, especially the brain stem using the olfactory pathway.

Damage to neuronal tissue occurs mainly by two mechanism i.e. immune mediated damage to the CNS and hypoxic injury. The cytokine storm in the brain tissue causes an increase in the level of inflammatory cytokines and activates endothelial cells, macrophages, and Tlymphocytes. This leads to increased concentration of IL-6 which activates complement and coagulation pathways, disseminated intravascular coagulation (DIC) and organ failure. On the other hand, the hypoxic brain injury due to severe pneumonia results in neuronal inflammation and brain edema [9].

\section{EPIDEMIOLOGY OF COVID-19 AND NEUROLOGICAL MANIFESTATIONS}

It is known that HCoVs invade neurological tissue via immune escape where they infect neurons resulting in neurological disorders and neurodegeneration. These findings are supported by the observations during the 20022003 pandemic of SARS-CoV. The autopsies examinations during the pandemic revealed the presence of $\mathrm{HCoV}_{\mathrm{s}}$ particles in the infected neuronal tissues. In the past, 
outbreaks of other coronaviruses like SARS-CoV and MERS-CoV have also been related to neurological manifestations. A study of a patient with SARS-CoV showed that the patient developed neurological manifestations on the 28th day of infection. As the genomic sequence of SARS-CoV-2 is $96 \%$ similar to the bat SARS$\mathrm{CoV}$, it has been reported that SARS-CoV-2 also leads to neurological manifestations in patients with COVID-19.

COVID-19 patients have most commonly an impaired sense of taste or smell or both. The MRI and CT scan of a patient experiencing dysgeusia and anosmia showed the obstruction of olfactory clefts due to bilateral inflammation, causing the loss of hair-like endings of the olfactory cell receptors making them unable to pick up odor molecules. A study conducted by Xydakis et al. [14], reported the occurrence of only anosmia, or in combination with dysgeusia is commonly encountered in COVID-19 patients. This can be attributed to the genomic and structural similarities between SARS-CoV-2 and SARS-CoV [11]. The loss of sense of taste and smell in COVID-19 affected individuals along with neurological signs and symptoms is considered to be result of viral entry into the CNS via the cribriform plate of the ethmoid bone that is in proximity to the olfactory bulb. This route of viral entry is generally considered in the case of early phase infected patients [8].

Another study evaluated the impaired sense of smell using a questionnaire, Sino-Nasal Outcome Test 22 (SNOT22 ), in patients with mild SARS-CoV-2 infection. They reported that out of 202 patients with positive nasopharyngeal RT-PCR for SARS-CoV-2, 130 patients experienced mild to moderate impairment in the sense of smell for 14 days prior to RT-PCR testing [15]. In adults, post viral anosmia that is secondary to the initial congestion of nose, is one of the main causes of loss of sense of smell. An online survey using questionnaire was conducted in the USA on 10,069 COVID-19 patients. The survey found that all of the patients experienced dysfunction in the sense of smell within the last 4 weeks after COVID-19 infection. The loss of sense of smell was reported in $48.23 \%$ of patients and the dysfunctional sense of taste was reported in $83.38 \%$ of patients. $76.24 \%$ patients developed anosmia suddenly after SARS-CoV-2 infection. Before developing anosmia, the participants experienced symptoms of flu or cold. Another cross-sectional survey was conducted online in USA to document chemosensory dysfunction in COVID-19 positive and COVID-19 negative patients. The loss of sense of smell (68\% Vs. 16\%) and taste (71\% Vs. 17\%) was higher in positive COVID-19 patients as compared to negative COVID-19 patients. The patients involved in the study were mostly ambulatory and did not require hospitalization. They interpreted that SARS-CoV-2 infection spreads through the nasal route in ambulatory patients while the infection spreads through the pulmonary route in severely ill-patients [9].

Recently, there is a case report of viral encephalitis in a middle-aged-man with COVID-19. the patient had a positive CSF RT-PCR for SARS-CoV-2 and normal CT scan of skull [16]. There are also reports of self-limiting encephalitis in COVID-19 patients. A patient presented with symptoms of fever, myalgia, and neurological symptoms while the patient IgG and IgM were negative for CSF [17]. Another case study reported that meningoencephalitis, is a major clinical manifestation in patients infected from SARS-CoV-2. The patient presented with meningoencephalitis without developing respiratory symptoms [18]. Acute myelitis has also been reported in a 66-year-old man with initial symptoms of fever and body pain. On admission, he developed sensory level at T-10, acute flaccid paralysis of lower limbs, urinary and bowel incontinence [19].

Acute necrotizing hemorrhagic encephalopathy (ANE) is also a complication of COVID-19 in severely-ill patients [3]. A female patient of COVID-19 in her late 50s, presented with a history of fever, cough and altered mental condition. Her brain MRI showed hemorrhagic rim of enhancing lesions within the medial temporal lobes, subinsular regions, and bilateral thalami. The CT images of her head revealed bilateral hypo-attenuation symmetrically within the medial thalami with a normal CT venogram and normal CT angiogram. Another cohort study in France reported three cases of ischemic strokes in COVID-19 patients. The patients did not develop focal neurological signs. The neuroimaging of the patients for encephalopathy revealed ischemic strokes. The absence of neurological symptoms was observed due to the presence of encephalopathy [9]. It is thought to be the result of intracranial cytokine storm. The cytokine storm causes the breakdown of blood-brain barrier without para-infectious demyelination or direct viral invasion. The acute necrotizing hemorrhagic syndrome has also been observed as a rare complication of influenza and other viral infections. The cytokine storm in severe COVID-19 patients resembles the profile of secondary hemophagocytic lymphohistiocytosis (HLH), characterized by increased levels of granulocytecolony stimulating factor (G-CSF),tumor necrosis factoralpha (TNF- $\alpha)$, interferon-alpha (IF- $\alpha)$, macrophage inflammatory protein 1-alpha, monocyte chemoattractant protein 1, IL-2, and IL-7 [3].

Guillain-Barre syndrome (GBS) has also been reported in COVID-19 patients. A study from Wuhan, China reported the symptoms of Guillain-Barre syndrome in a 61-year old female. The patient had a travel history to Wuhan, China, but she was asymptomatic for COVID-19. Later, she developed dry cough ad fever, and was tested positive for COVID-19, during follow-up treatment. The GBS was reversible and she recovered after treatment [20]. Similar cases of GBS were also observed in a cohort study of 23 patients infected with MERS-CoV [21]. GBS has also been observed in 5 patients of COVID-19 from Italy. Four patients presented with paresthesia and lower limb weakness, followed by ataxia and facial weakness, while one patient presented with paresthesia only. The Electromyography (EMG) of 3 patients showed axonal polyneuropathy while the other 2 patients developed demyelinating polyneuropathy [22].

Another patient from China initially developed signs of autoimmune neuropathy and later tested positive for SARSCoV-2 infection [3]. It was suggested that SARS-CoV-2 is responsible for this condition due to its temporal association with Guillain-Barre syndrome (GBS)/Acute Inflammatory Demyelinating Polyneuropathy (AIDP). The occurrence of polyneuritis cranialis and Miller Fischer syndrome have also been reported in two cases of COVID-19 in Spain. The 
peripheral demyelination is a result of precipitating demyelination secondary to the neuro-invasive nature of the virus that trigger a hyperactive immune response due to virus induced inflammatory response [3].

In severe cases of COVID-19, the high D-dimer level leads to stroke or cerebrovascular accident. The viral entry in the cerebral circulation causes rupture in endothelium and thrombus formation. The slow cerebral circulation favors the increase viral replication and endothelial rupture. Also, the elevated D-dimer level alters the coagulation cascade which further leads to stroke [3]. Cerebrovascular accident due to intracranial bleed was reported in a 79-year-old patient of COVID-19 in Iran. The patient was admitted in emergency with a history of cough and fever. His chest CT scan revealed ground glass opacity and the brain CT scan showed massive bleed in the right cerebral hemisphere with subarachnoid and intraventricular extension. It was postulated that bleed could be the result of dysregulation of ACE2 receptors leading to sympatho-adrenal system, cerebral auto-regulation and blood flow.

A study in China reported that $40 \%$ of COVID-19 patients develop encephalopathy and headache. Another study of 113 COVID-19 patients reported that 20 patients developed hypoxic encephalopathy during the illness. The incidence of minor symptoms including headache and dizziness, is $3-12.1 \%$ in COVID-19 patients. Neuralgia, ataxia and epilepsy has also been reported in COVID-19 patients [9].

\section{CONCLUSION}

Neurological manifestations are also common in COVID19 patients and if untreated, can be fatal. A differential diagnosis of neurological manifestations and complications is required for the proper treatment and good prognosis of patients. There is further needed to understand the neuroinvasive nature of SARS-CoV-2 and to develop proper diagnostic tests and treatment for neurological disorders of COVID-19.

\section{REFERENCES}

[1] Zhao, Y., Zhao, Z., Wang, Y., Zhou, Y., Ma, Y., \& Zuo, W. (2020). Single-Cell RNA Expression Profiling of ACE2, the Putative Receptor of Wuhan 2019-nCov. BioRxiv. https://doi.org/10.1101/2020.01.26.919985.

[2] Guan, W., Ni, Z., Hu, Y., Liang, W., Ou, C., He, J., Zhong, N. (2020) Clinical characteristics of 2019 novel coronavirus infection in China. MedRxiv. https://doi.org/10.1101/2020.02.06.20020974.

[3] Jasti, M., Nalleballe, K., Dandu, V., \& Onteddu, S. (2020). A review of pathophysiology and neuropsychiatric manifestations of COVID19. Journal of Neurology, (0123456789). https://doi.org/10.1007/s00415-020-09950-w.

[4] Mao, L., Jin, H., Wang, M., Hu, Y., Chen, S., He, Q., Hu, B. (2020). Neurologic Manifestations of Hospitalized Patients with Coronavirus Disease 2019 in Wuhan, China. JAMA Neurology. https://doi.org/10.1001/jamaneurol.2020.1127.

[5] Xu, H., Zhong, L., Deng, J., Peng, J., Dan, H., Zeng, X., Chen, Q. (2020). High Expression of ACE2 Receptor of 2019-nCoV on the Epithelial Cells of Oral Mucosa. International Journal of Oral Science. https://doi.org/10.1038/s41368-020-0074-x.

[6] Baig, A. M., Khaleeq, A., Ali, U., \& Syeda, H. (2020). Evidence of the COVID-19 Virus Targeting the CNS: Tissue Distribution, Host Virus Interaction, and Proposed Neurotropic Mechanisms. ACS Chemical https://doi.org/10.1021/acschemneuro.0c00122.
[7] Conde Cardona, G., Quintana Pájaro, L. D., Quintero Marzola, I. D., Ramos Villegas, Y., \& Moscote Salazar, L. R. (2020). Neurotropism of SARS-CoV 2: Mechanisms and manifestations. Journal of the Neurological Sciences. https://doi.org/10.1016/j.jns.2020.116824.

[8] Baig, A. M. (2020). Neurological manifestations in COVID-19. CNS Neuroscience \& Therapeutics. https://doi.org/10.1111/cns.13372.

[9] Ahmad, I., \& Azam, F. (2020). Neurological manifestations and complications of COVID-19: A literature review. Journal of Clinical Neuroscience. https://doi.org/10.1016/j.jocn.2020.05.017.

[10] Helms, J., Kremer, S., Merdji, H., Clere-Jehl, R., Schenck, M., Kummerlen, C., Meziani, F. (2020). Neurologic features in severe SARS-COV-2 infection. New England Journal of Medicine. https://doi.org/10.1056/NEJMc2008597.

[11] Acharya, A., Kevadiya, B. D., Gendelman, H. E., \& Byrareddy, S. N (2020). SARS-CoV-2 Infection Leads to Neurological Dysfunction. Journal of Neuroimmune Pharmacology. https://doi.org/: 10.1007/s11481-020-09924-9.

[12] Li, Y. C., Bai, W. Z., \& Hashikawa, T. (2020). The Neuroinvasive Potential of SARS-CoV2 May Play a Role in the Respiratory Failure of COVID-19 Patients. Journal of Medical Virology. https://doi.org/10.1002/jmv.25728.

[13] Poyiadji, N., Shahin, G., Noujaim, D., Stone, M., Patel, S., \& Griffith, B. (2020). COVID-19-Associated Acute Hemorrhagic Necrotizing Encephalopathy: CT and MRI Features. Radiology. https://doi.org/10.1148/radiol.2020201187.

[14] Xydakis M.S., Dehgani-Mobaraki, P., Holbrook, E.H., Geisthoff, U.W., Bauer, C., Hautefort, C., Herman, P., Manley, G.T., Lyon, D.M., \& Hopkins, C. (2020). Smell and taste dysfunction in patients with COVID-19. The Lancet Infectious Diseases. https://doi: 10.1016/S1473-3099(20)30293-0

[15] Spinato, G., Fabbris, C., Polesel, J., Cazzador, D., Borsetto, D., Hopkins, C., \& Boscolo-Rizzo, P. (2020). Alterations in smell or taste in mildly sympotomatic outpatients with SARS-CoV-2 infection JAMA, https://doi.org/10.1001/jama.2020.6771.

[16] Moriguchi, T., Harii, N., Goto, J., Harada, D., Sugawara, H., Takamino, J., Shimada, S. (2020). A First Case of Meningitis/Encephalitis Associated with SARS-Coronavirus-2. International Journal of Infectious Diseases. https://doi.org/10.1016/j.ijid.2020.03.062.

[17] Ye, M., Ren, Y., \& Lv, T. (2020). Encephalitis as a Clinical Manifestation of COVID-19. Brain, Behavior, and Immunity. https://doi.org/10.1016/j.bbi.2020.04.017.

[18] Duong, L., Xu, P., \& Liu, A. (2020). Meningoencephalitis without respiratory failure in a young female patient with COVID-19 infection in Downtown Los Angeles, early April 2020. Brain, Behavior, and Immunity. https://doi.org/10.1016/j.bbi.2020.04.024.

[19] Zhao, K., Huang, J., Dai, D., Feng, Y., Liu, L., \& Nie, S. (2020) Acute Myelitis after SARS-CoV-2 Infection: A Case Report. MedRxiv. https://doi.org/10.1101/2020.03.16.20035105.

[20] Zhao, H., Shen, D., Zhou, H., Liu, J., \& Chen, S. (2020). GuillainBarré Syndrome Associated with SARS-CoV-2 Infection: Causality or Coincidence? The Lancet Neurology https://doi.org/10.1016/S1474-4422(20)30109-5

[21] Kim, J. E., Heo, J. H., Kim, H. O., Song, S. H., Park, S. S., Park, T. H., Choi, J. P. (2017). Neurological complications during treatment of middle east respiratory syndrome. Journal of Clinical Neurology (Korea). https://doi.org/10.3988/jcn.2017.13.3.227.

[22] Toscano, G., Palmerini, F., Ravaglia, S., Ruiz, L., Invernizzi, P., Cuzzoni, M. G., Micieli, G. (2020). Guillain-Barré Syndrome Associated with SARS-CoV-2. New England Journal of Medicine. https://doi.org/10.1056/nejmc2009191. 